\title{
Parámetros genéticos de rasgos biométricos del fruto y semilla en Benincasa hispida (Thunb.) Cogn.
}

\author{
Miguel M. Espitia-Camacho, Hermes Araméndiz-Tatis y Carlos E. Cardona-Ayala \\ Facultad de Ciencias Agrícolas. Universidad de Córdoba, Carrera 6 No. 77- 305 - Montería - Colombia. \\ (Correo-e: mmespitia@correo.unicordoba.edu.co; haramendiz@correo.unicordoba.edu.co; \\ cecardona@correo.unicordoba.edu.co).
}

Recibido Ene. 12, 2021; Aceptado Mar. 15, 2021; Versión final Abr. 12, 2021, Publicado Ago. 2021

\begin{abstract}
Resumen
El objetivo de esta investigación fue cuantificar los parámetros genéticos de 16 características de frutos y semillas de Benincasa hispida (Thunb.) Cogn. en Montería (Colombia). Se evaluaron 10 familias de hermanos medios, en un diseño completamente aleatorizado con cinco repeticiones. Se detectaron diferencias sólo entre familias de hermanos medios para ancho de semilla. Las estimaciones de variabilidad genética fueron mayores en ancho y grosor de semillas, donde el componente genético representó el $81,58 \%$ y $45,91 \%$ de la varianza fenotípica total. Las mayores heredabilidades en sentido amplio $\left(\mathrm{h}^{2} \mathrm{~A}=81,58 \%\right)$ y estricto $\left(\mathrm{h}^{2} \mathrm{E}=\right.$ $13,05 \%$ ) se presentaron en ancho de semillas. La ganancia genética esperada expresada como porcentaje de la media, ocurrió en ancho y volumen de semilla, con progreso $60 \%$ superior a la media general. Se concluyen que los parámetros genéticos obtenidos indican que la selección de frutos con semillas anchas, gruesas y voluminosas, permitirán obtener plántulas vigorosas para propagación.
\end{abstract}

Palabras clave: varianza genética; heredabilidad; ganancia; selección; mejoramiento

\section{Genetic parameters of Benincasa hispida (Thunb.) Cogn. fruit and seed biometric traits}

\begin{abstract}
The main objective of this research study was to quantify the genetic parameters of 16 fruit and seed traits of Benincasa hispida (Thunb.) Cogn from Montería (Colombia). Ten families of half siblings (FHM) were evaluated in a completely randomized design with five replications. The results showed differences only between FHM for seed width (SW). The estimates of genetic variability were higher in SW and seed thickness, where the genetic component represented $81.58 \%$ and $45.91 \%$ of the phenotypic variance. The greatest heritability in broad sense $\left(\mathrm{h}^{2} \mathrm{~A}=81.58 \%\right)$ and strict sense $\left(\mathrm{h}^{2} \mathrm{E}=13.05 \%\right)$ occurred in SW. Expected genetic gain expressed as a percentage of the mean occurred in SW and seed volume, being $60 \%$ higher than that of the general mean. In conclusion, the genetic parameters obtained here indicate that selecting fruits with broad, thick, and voluminous seeds would allow obtaining vigorous seedlings for propagation.
\end{abstract}

Keywords: genetic variance; heritability; gain; selection; improvement 


\section{INTRODUCCIÓN}

La especie $B$. hispida, $(2 \mathrm{n}=2 \mathrm{x}=24)$, conocida como pepino chino o calabaza de ceniza, es una cucurbitácea, monoica, alógama, que se cultiva principalmente desde el Sur y Este de Asia (Al-Snafi, 2013; Ekeke et al., 2019; Chomicki et al., 2020); donde goza de una gran importancia en la alimentación, nutrición, biomedicina, medicina tradicional e industria farmacológica (Jiang et al., 2016), bebidas, charcutería, delicatesen, artesanías, tradiciones culturales y religiosas, entre otras (Chakraborty et al., 2018; Gupta et al., 2019; Chomicki et al., 2020). Los frutos pueden ser cilíndricos, oblongos o redondeados, dependiendo del cultivar; provistos de una gruesa cera en la epidermis cuando maduran, llegando a pesar hasta 13,67 kg (Pandey et al., 2015; Tadkal et al., 2019). Las semillas, ubicadas en el centro del fruto, son ovales-elípticas, aplanadas, lisas y de color amarillo claro, miden entre 1,0 y $1,5 \mathrm{~cm}$ de largo y 0,5 a 0,8 cm de ancho (Tadkal et al., 2019; Pradhan et al., 2020). B. hispida es de utilidad en las economías campesinas y en los cultivos de pancoger, pero muy poco investigadas en cuanto a la conservación de su germoplasma, identificación e inventario de ecotipos, obtención y producción de semillas, desarrollo de paquetes tecnológicos, cosecha, postcosecha y transformación en general (Ekeke et al., 2019; Gupta et al., 2019; Tadkal et al., 2019).

El conocimiento de los parámetros genéticos de las principales características de interés agronómicos en las poblaciones sometidas a selección es de gran importancia para el mejorador, ya que son esenciales para definir la estructura genética de las poblaciones, hacer control genético de las características, inferir su variabilidad genética y orientar la elección del mejor método de mejoramiento para la población, que maximice las ganancias genéticas por selección (Araméndiz et al., 2016; Lovely y Vijayaraghava, 2017; Pradhan et al., 2018). La efectividad de la selección en cualquier programa de mejoramiento genético de plantas, depende principalmente de la variabilidad genética aditiva presente en la población. La heredabilidad y el avance genético son parámetros de selección importantes. El estudio de la variabilidad genética, heredabilidad y avance genético de diferentes rasgos del acervo genético, facilitará la evaluación e identificación de genotipos superiores (Sureja et al., 2010; Tasiguano et al., 2019). Las estimaciones de heredabilidad ayudan en la selección de genotipos élite de diversas poblaciones genéticas. El avance genético mide la cantidad de progreso que se podría esperar con la selección. En la mejora de poblaciones solo el componente genético aditivo se transmite a la siguiente generación. El grado de mejora depende además de la intensidad de la selección y el avance genético obtenido de la población (Lovely y Vijayaraghava, 2017; Pradhan et al., 2018).

Estudios de parámetros genéticos en B. hispida han sido reportados por Nagaraju at al. (2016); Lovely y Vijayaraghava (2017); Pradhan et al. (2018), con resultados variables en la magnitud de las estimaciones de los parámetros más importantes, como variabilidad genética, heredabilidad y progreso genético por selección directa e indirecta; no obstante, muchos de ellos han permitido progresos genéticos significativos en la conservación, uso y mejora de los caracteres de interés e igualmente, han coadyuvado al desarrollo y obtención de poblaciones genéticamente superiores, con mayor adaptación, producción, calidad y productividad de cultivares, de acuerdo a las necesidades, condiciones y requerimientos de los productores, transformadores y consumidores finales.

En Colombia, a pesar de que existen, se cultivan e investigan varias especies de la familia cucurbitaceae y otras especies (Rodríguez-Manrique et al., 2018; Estrada at al., 2018), B. hispida es relativamente nueva, ha sido subutilizada y poco o nada investigada, por tanto, se desconoce la variabilidad genética y heredabilidad de las características de interés de la especie, entre otros. Esta situación justificó la necesidad de investigar los parámetros genéticos del fruto y sus semillas, para aumentar su conocimiento y aprovecharlo en la conservación de germoplasma e inventario de genotipos, además de su uso sostenible en programas de mejoramiento genético de la especie, para mejorar poblaciones y obtener cultivares para el mercado nacional. El objetivo del presente trabajo fue estimar los parámetros genéticos para características biométricas del fruto y semilla de $B$. hispida, en Montería (Colombia), como información básica para futuros programas de mejoramiento y conservación genética de la especie.

\section{METODOLOGÍA}

La metodología se presenta atendiendo los componentes más importantes relacionados con los materiales y métodos utilizados en el estudio: Localización y duración, material genético, variables de respuestas, diseño experimental y análisis de datos.

\section{Localización y duración}

El estudio de campo y laboratorio se realizó entre agosto/2016 y julio/2017, en un lote experimental (plano, uniforme, aluvial y perteneciente a la zona agroecológica Cj) y en el Laboratorio de Genética Vegetal y Fitomejoramiento (LAGEFI) de la Universidad de Córdoba (Montería - Colombia), ubicada en la zona media del valle del Sinú, a $8^{\circ} 52^{\prime}$ de latitud norte y $76^{\circ} 48^{\prime}$ longitud oeste, a una altura de $13 \mathrm{msnm}$. La zona ecológica 
corresponde al bosque seco tropical con temperatura promedio de $28{ }^{\circ} \mathrm{C}$, humedad relativa de $84 \%$ y precipitación anual de $1200 \mathrm{~mm}$ (Palencia et al., 2006).

\section{Material genético}

La investigación se realizó mediante la siembra de un ensayo de evaluación agronómica de 10 familias de hermanos medios (FHM), con cinco repeticiones. Las $10 \mathrm{FHM}$ se originaron de 10 plantas seleccionadas fenotípicamente de un lote semi-comercial de 0,5 ha conocida como "pepino chino", sembrado en el primer semestre de 2016, en la granja experimental de la Universidad de Córdoba. La semilla utilizada para el estudio se obtuvo de un compuesto balanceado de los frutos frescos, completos, sanos, maduros y de libre polinización de cada familia seleccionada.

\section{Variables de respuestas}

Al momento de la cosecha del ensayo se estimaron 16 características biométricas del fruto y semillas de $B$. hispida. En cada unidad experimental se seleccionaron al azar cinco frutos de libre polinización, a los cuales se les midieron ocho rasgos biométricos: largo máximo (LF), ancho máximo (AF), grosor o diámetro máximo (GF), grosor de la pulpa (GPF) y cavidad de la semilla (CSF), con la ayuda de reglas y cintas métricas metálicas marcadas a escala de centímetros-milimetradas. Se utilizó una balanza de precisión marca BJ 410C, para estimar en gramos el peso fresco/fruto (PF), el peso de las semillas/fruto (PESF) y el número de semillas/fruto (NSF).

Para los rasgos de la semilla de cada fruto fueron seleccionadas 50 semillas que se mezclaron para formar un compuesto balanceado, a las cuales se les estimaron ocho variables biométricas: el ancho máximo de una semilla (AS), el largo máximo (LS) y el grosor máximo (GS), empleando un nonio Spictools $\circledast$. Asimismo, el peso de una semilla (P1S), el peso de cien semillas (P100S), el número de semillas por kilogramo (NSK), utilizando una balanza BJ 410C, el volumen de una semilla (V1S) se estimó como el incremento volumétrico $(\mathrm{ml})$, generado en una probeta con volumen de agua destilada conocido, al introducir una muestra de 100 semillas tomadas al azar por cada unidad experimental de cada FHM y la densidad de una semilla (D1S) fue estimada a partir de la relación (P1S/V1S).

\section{Diseño experimental y análisis de datos}

Para la evaluación de características morfométricas de las 10 familias se utilizó el diseño completamente aleatorizado con cinco repeticiones y tamaño de unidades experimentales de tres surcos por ocho metros de largo, con distancias de siembra de $2 \mathrm{~m}$ por $2 \mathrm{~m}$ en cuadro. Se realizaron análisis de varianza para estimar la variación entre y dentro de familias o poblaciones. Además, se estimaron los parámetros genéticos: coeficientes de variación fenotípicos (CVF), varianza fenotípica media entre familias de hermanos medios (VF), varianza genética media entre familias de hermanos medios (VG), varianza ambiental media entre familias de hermanos medios (VA), varianza genética aditiva media entre familias de hermanos medios (VGA), coeficientes de variación genotípicos (CVG), heredabilidad en sentido amplio $\left(\mathrm{h}^{2} \mathrm{~A}\right)$, heredabilidad en sentido estricto $\left(\mathrm{h}^{2} \mathrm{E}\right)$, Correlación intraclase (CORI), relación entre $\mathrm{CVG} / \mathrm{CVe}(\mathrm{b})$ y estadísticas descriptivas para las 16 variables de respuesta consideradas. Los análisis estadísticos y parámetros genéticos se realizaron mediante el uso del programa computacional de libre acceso GENES versión Windows GENES V.2014.6.1 (Cruz, 2016). Los parámetros genéticos fueron estimados con el programa GENES a partir de los resultados del análisis de varianzas y teniendo en cuenta los cuadrados medios esperados (Tabla 1).

La heredabilidad en sentido amplio $\left(h^{2} A\right)$ y estricto $\left(h^{2} E\right)$ para cada variable, fue estimada de la forma clásica, como se describe en las ecuaciones 1 y 2 . En estas ecuaciones, $\sigma^{2} G$ : varianza genética media para familias de hermanos medios (HM); $\sigma^{2} \mathrm{~F}$ : Varianza fenotípica media para familias de $\mathrm{HM} ; \sigma^{2} \mathrm{~A}$ : Varianza genética aditiva media para familias de HM.

$$
\begin{aligned}
& h_{A}^{2}=\left(\frac{\sigma_{G}^{2}}{\sigma_{F}^{2}}\right) \times 100 \\
& h_{E}^{2}=\left(\frac{\sigma_{A}^{2}}{\sigma_{F}^{2}}\right) \times 100
\end{aligned}
$$

En la Tabla 1, f: es el número de familias de hermanos medios $=10 ;$ r: repeticiones $=5 ; \mathrm{CM}_{\mathrm{H}}$ : Cuadrados medios para familias de hermanos medios $(\mathrm{HM}) ; \mathrm{CM}_{\mathrm{E}}$ : Cuadrados medio del error o residual; $\sigma^{2} \mathrm{HM}=$ varianza media entre familias de $\mathrm{HM} ; \sigma^{2} \mathrm{HM}=\sigma^{2} \mathrm{G}=$ varianza genética $=\left(\mathrm{CM}_{\mathrm{HM}}-\mathrm{CM}_{\mathrm{E}}\right) / \mathrm{r} ; \sigma^{2}=$ varianza ambiental de

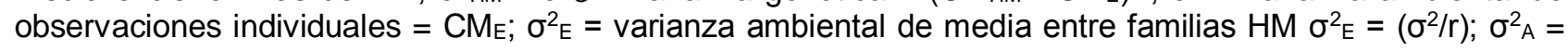


varianza aditiva media $=\left(4 \sigma^{2} \mathrm{HM} / \mathrm{r}\right) ; \sigma^{2} \mathrm{~F}=$ varianza fenotípica de medias entre familias de $\mathrm{HM}=\sigma^{2} \mathrm{~F}=\left(\sigma^{2} \mathrm{G}+\right.$ $\left.\sigma^{2} \mathrm{E}\right)$. (Hallauer et al., 1988).

Tabla 1. Fuentes de variación (FV), grados de libertad (GL) y cuadrados medios (CM) esperados para el análisis de varianza, bajo el uso del diseño completamente al azar con 10 tratamientos y 5 repeticiones.

\begin{tabular}{|l|c|c|c|}
\hline \multicolumn{1}{|c|}{$F V$} & $G L$ & $C M$ & $C M$ Esperados \\
\hline Familias HM & $(\mathrm{f}-1)=9$ & $\mathrm{CM}_{\mathrm{HM}}$ & $\sigma^{2}+r \sigma^{2} \mathrm{HM}$ \\
\hline Error & $\mathrm{f}(\mathrm{r}-1)=40$ & $\mathrm{CM}_{\mathrm{E}}$ & $\sigma^{2}$ \\
\hline Total & $(\mathrm{rf}-1)=49$ & & \\
\hline
\end{tabular}

La ganancia genética esperada (GGE) para cada variable, se estimó a través de la ecuación 3, propuesta por Hallauer et al. (1988). Donde $k=$ Diferencial de selección al $5 \%$ de intensidad de selección, equivalente a 2,06; $\sigma_{F}=$ Desviación estándar fenotípica; $h^{2} E=$ Heredabilidad en sentido estricto. La GGE también se expresó en porcentaje GGE (\%) de la media general, de acuerdo con la ecuación 4. El rango de la GGE (\%) fue clasificado, como: bajo $(<10 \%)$, moderado $(10-20 \%)$ y alto $(>20 \%)$.

$\mathrm{GGE}=\mathrm{k} \times \sigma_{\mathrm{F}} \times \mathrm{h}_{\mathrm{E}}^{2}$

GGE $(\%)=($ GGE/Media $) \times 100$

La presencia de una sequía, daños en el sistema de riego por microaspersión, pérdida de población en las unidades experimentales, obligaron a descartar la segunda evaluación (2017B) y no contar con información de campo suficiente para estimar los efectos de interacción FHM X Semestre y parámetros de forma más precisa. Por ello se sugiere tomar los resultados como preliminares y con cierta precaución.

\section{RESULTADOS}

Los resultados se presentan atendiendo el orden del análisis secuencial lógico en estudios de parámetros genéticos en plantas, cuando se evalúan varias poblaciones con estructura de familias de hermanos medios (FHM) con el uso del diseño experimental y varias repeticiones por FHM.

\section{Análisis de varianza para las características biométricas}

Los cuadrados medios de los análisis de varianza (Tabla 2), permitieron detectar diferencias altamente significativas $(p<0,01)$ entre FHM sólo para la característica AS, más no así en las 15 variables restantes: PF, LF, AF, GF, GPF, CSF, PESF, NSF, LS, GS, P1S, P100S, NSK, V1S y D1S.

Tabla 2. Cuadrados medios y nivel de significancia para 16 características biométricas del fruto y semillas de $B$. hispida en Montería (Colombia)

\begin{tabular}{|c|c|c|c|c|}
\hline \multirow{2}{*}{ Variable } & \multicolumn{2}{|c|}{ Cuadrados medios } & \multirow{2}{*}{ Media } & \multirow{2}{*}{ CV (\%) } \\
\cline { 2 - 3 } & Tratamientos $(g l=9)$ & Error $(g l=40)$ & & \\
\hline PF & $371370,81 \mathrm{~ns}$ & 359294,7 & 3334,12 & 17,98 \\
\hline LF & $5,55 \mathrm{~ns}$ & 3,77 & 24,47 & 7,93 \\
\hline AF & $1,75 \mathrm{~ns}$ & 2,51 & 17,16 & 9,23 \\
\hline GF & $2,94 \mathrm{~ns}$ & 1,82 & 17,02 & 7,93 \\
\hline GPF & $0,16 \mathrm{~ns}$ & 0,12 & 3,38 & 10,25 \\
\hline CSF & $1,84 \mathrm{~ns}$ & 1,48 & 10,48 & 11,61 \\
\hline PESF & $115,61 \mathrm{~ns}$ & 145,73 & 47,71 & 25,30 \\
\hline NSF & $89181,61 \mathrm{~ns}$ & 94455,10 & 1265,90 & 24,28 \\
\hline LS & $0,00283 \mathrm{~ns}$ & 0,00216 & 1,03 & 4,51 \\
\hline GS & $0,00009 \mathrm{~ns}$ & 0,00005 & 0,16 & 4,42 \\
\hline AS & 0,00585 ** & 0,00108 & 0,57 & 5,77 \\
\hline
\end{tabular}


Tabla 2: continuación.

\begin{tabular}{|c|c|c|c|c|}
\hline \multirow{2}{*}{ Variable } & \multicolumn{2}{|c|}{ Cuadrados medios } & \multirow{2}{*}{ Media } & \multirow{2}{*}{ CV (\%) } \\
\cline { 2 - 3 } & Tratamientos $(g l=9)$ & Error $(g l=40)$ & & \\
\hline P1S & $0,000027 \mathrm{~ns}$ & 0,00002 & 0,03 & 14,91 \\
\hline P100S & $0,1369 \mathrm{~ns}$ & 0,1191 & 3,78 & 9,13 \\
\hline NSK & $7596955,01 \mathrm{~ns}$ & 5478913,52 & 26631,96 & 8,79 \\
\hline V1S & $0,00024 \mathrm{~ns}$ & 0,00016 & 0,05 & 25,30 \\
\hline D1S & $0,0303 \mathrm{~ns}$ & 0,0252 & 0,80 & 19,84 \\
\hline
\end{tabular}

En la Tabla 2: ns: es no significativo al 5\%, ${ }^{* *}$ : significativo al 1\%; CV (\%): coeficiente de variación; PF: peso fresco / fruto; LF: largo máximo / fruto; AF: ancho máximo / fruto; GF: grosor o diámetro máximo / fruto; GPF: grosor de la pulpa / fruto; CSF: cavidad de la semilla / fruto; PESF: peso de la semilla / fruto; NSF: número de semillas / fruto; LS: largo de semilla; GS: grosor de la semilla; AS: ancho de semilla; P1S: peso de una semilla; P100S: peso de 100 semillas; NSKG: número de semillas / kilogramo; V1S: volumen de una semilla; D1S: densidad de una semilla.

\section{Parámetros genéticos}

Los parámetros genéticos derivados de las características biométricas del fruto y las semillas de $B$. hispida se encuentran registrados en la Tabla 3. Los caracteres biométricos GS, LS, AS, GF, LF, NSK, P100S y AF, sobresalieron por presentar los más bajos CVF, menores del 10\%; a su vez las variables GPF, CSF, P1S y PF, con CVF entre el 10,25\% y 17,98\%, presentaron dispersión media; mientras que D1S, NSF, PESF y V1S, exhibieron la mayor heterogeneidad y dispersión, por los más altos CVF del 19,84\% al 25,30\% (Tabla 2). En todos los caracteres considerados, las estimaciones promedio de las VG, en general fueron bajos, excepto para el AS y GS de las semillas, en donde el componente genético representó el 81,58\% y 45,91\% de la varianza fenotípica. Lo anterior, se complementa con valores altos de VA para la mayoría de los caracteres estudiados.

Al comparar los CVF de los 16 caracteres biométricos de los frutos y semillas, con sus correspondientes CVG, se detecta un agrupamiento de los caracteres por su magnitud diferente, ya que en general los CVG fueron menores del $9 \%$, con valores de VG y VGA negativos, (asumidos como cero) o positivos, muy cercanos a cero $(<3 \%)$ para la mayoría de las características, exceptuando AS, D1S y P1S, las cuales presentaron los más altos CVG, con valores de 4,01\%, 5,36\% y 8,11\%, respectivamente (Tabla 3). Así mismo, en general se observaron bajos índices de b con valores menores a 0,39, excepto para el AS, que presentó un valor de 0,93 .

La mayor estimación de heredabilidad $\left(\mathrm{h}^{2} \mathrm{~A}\right)$ y $\left(\mathrm{h}^{2} \mathrm{E}\right)$ se encontró para el AS, con valores de $81,58 \%$ y $13,05 \%$, respectivamente. Las 16 variables se agruparon como de $\mathrm{h}^{2} \mathrm{~A}$ alta $(>50 \%)$ : AS; heredabilidad media $(20-$ $50 \%$ ): GS, GF, V1S, LF, NSK, P1S, GPF y LS; heredabilidad baja (<20\%): CVS, D1S, P100S, PF, NSF, PESF y AF. Según los valores de $h^{2}$ E, todas las variables se clasificaron como de heredabilidad baja $(<20 \%)($ Tabla 3). La ganancia genética esperada expresada como GGE\% de las $10 \mathrm{FHM}$ evaluados, fue importante sólo en dos de los 16 caracteres estudiados, AS y V1S, con aproximadamente un incremento en la GGE (\%) del 60\% superior a sus respectivas medias, mientras que el resto de las variables, se agruparon como de baja GGE (\%) por selección, dado que los valores obtenidos no superaron a la media general (Tabla 3).

En la Tabla 3, CVF: es el coeficiente de variación fenotípico; VF (Media): varianza fenotípica media entre familias de hermanos medios (HM); VA (Media): varianza ambiental media entre familias de HM; VG (Media): varianza genética media entre familias de HM; VGA (Media): varianza genética aditiva media entre familias de HM; CVG: coeficiente de variación genotípico; $h^{2}{ }^{A}$ : heredabilidad en sentido amplio; $h^{2}$ : heredabilidad en sentido estricto; CORI: correlación intraclase; b: relación. (CVG/CVe): índice de variabilidad; GGE: ganancia genética esperada; GGE(\%):ganancia genética esperada en \%; PEF: peso/fruto; LF: largo/fruto; AF: ancho/fruto; GF: grosor/fruto; GPF: grosor de la pulpa/fruto; CSF: cavidad de la semilla/fruto; PESF: peso de semilla/fruto; NSF: número de semillas/fruto; LS: largo de semilla; GS: grosor de semillas; AS: ancho de semilla; P1S: peso de una semilla; P100S: peso de 100 semillas; NSKG: número de semillas/kilogramo; V1S: volumen de una semilla; D1S: densidad de una semilla. Ind: Indeterminado. Los valores negativos se asumen como cero 


\begin{tabular}{|c|c|c|c|c|c|c|c|c|c|c|c|c|c|c|c|c|}
\hline 嵌ঃ & \begin{tabular}{l|l|}
$\infty$ \\
$\infty$ \\
$\infty$
\end{tabular} & $\begin{array}{l}0 \\
\dot{y} \\
y\end{array}$ & 웜 & $\mid \begin{array}{l}6 \\
5^{\circ}\end{array}$ & $\begin{array}{l}\infty \\
-\infty \\
-\end{array}$ & $\overline{\hat{m}}$ & $\begin{array}{l}\text { : } \\
\text { : }\end{array}$ & ํํㄹ & $\begin{array}{l}\circ \\
\infty \\
\infty\end{array}$ & $\mid \begin{array}{l}\infty \\
\infty \\
\infty \\
m\end{array}$ & m. & $\bar{g}$ & $\widehat{\stackrel{1}{0}}$ & 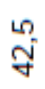 & $\overline{8}$ & $\begin{array}{l}6 \\
\dot{0}\end{array}$ \\
\hline 嵌 & $\begin{array}{l}\text { 을 } \\
\text { న్ } \\
\text { N. }\end{array}$ & $\begin{array}{l}\stackrel{5}{-} \\
=\end{array}$ & $\begin{array}{c}\bar{y} \\
0 \\
0\end{array}$ & $\begin{array}{l}8 \\
6 \\
\sigma\end{array}$ & $\underset{-}{-}$ & $\begin{array}{l}\infty \\
\mathbb{\infty}^{-}\end{array}$ & 部 & 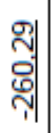 & $\frac{\sigma}{0}$ & $\begin{array}{l}\mathscr{0} \\
0\end{array}$ & $\begin{array}{l}\delta \\
\delta \\
\delta\end{array}$ & $\begin{array}{l}\bar{\delta} \\
\sigma^{\circ}\end{array}$ & $\begin{array}{c}\bar{T} \\
0 \\
0\end{array}$ & $\begin{array}{l}\text { L } \\
\text { స్ } \\
\text { = }\end{array}$ & $\begin{array}{l}0 \\
: \\
0\end{array}$ & 守 \\
\hline ــ & $\begin{array}{l}\infty \\
0 \\
0\end{array}$ & \begin{tabular}{|c|}
$\bar{m}$ \\
0 \\
0
\end{tabular} & 희 & $\mid \begin{array}{l}m \\
m \\
0 \\
0\end{array}$ & 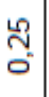 & $\begin{array}{l}\text { สี } \\
\text { - }\end{array}$ & 릐 & 희 & $\begin{array}{l}\stackrel{2}{0} \\
\stackrel{\circ}{\circ}\end{array}$ & 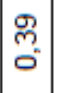 & $\begin{array}{l}\text { O. } \\
0 \\
0\end{array}$ & స్ & $\stackrel{\sim}{\circ}$ & $\begin{array}{l}\text { ָ̃ } \\
\text { ¿ }\end{array}$ & $\begin{array}{l}\tilde{m} \\
0 \\
0\end{array}$ & స్ \\
\hline 밍 & $\begin{array}{l}\hat{0} \\
0 \\
0\end{array}$ & $\mid \begin{array}{l} \\
0 \\
\infty\end{array}$ & 희 & $\mid \begin{array}{l}\mathscr{L} \\
0 \\
-\end{array}$ & $\begin{array}{l} \pm \\
0^{\circ}\end{array}$ & $\begin{array}{l}\stackrel{\circ}{0} \\
\forall \\
\forall\end{array}$ & 릐 & 희 & $\begin{array}{c}\infty \\
0 \\
\vdots \\
5\end{array}$ & $\mid \begin{array}{l}\bar{n} \\
\overline{0}\end{array}$ & $\begin{array}{l}\hat{s} \\
6 \\
\sigma \\
\end{array}$ & ఫ্ & $\stackrel{\text { S }}{\sim}$ & $\stackrel{\infty}{\Gamma}$ & $\begin{array}{l}\varphi \\
\sigma \\
\sigma\end{array}$ & $\begin{array}{l}\bar{\sim} \\
\sim\end{array}$ \\
\hline ঙૅఝ & $\mid \begin{array}{c}\mathrm{N} \\
0 \\
0\end{array}$ & $\frac{7}{50}$ & $\begin{array}{l}\text { क् } \\
\text { फी }\end{array}$ & $\frac{0}{6}$ & $\begin{array}{l}\mathscr{D} \\
\infty \\
\text { m. }\end{array}$ & $\bar{m}$ & 穵 & 네 & $\begin{array}{l}\stackrel{R}{2} \\
\hat{m}\end{array}$ & $\frac{\Sigma}{\sim}$ & $\begin{array}{l}\text { L } \\
\stackrel{m}{2}\end{array}$ & స్లి & $\begin{array}{l}\text { : } \\
\text { i }\end{array}$ & 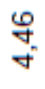 & 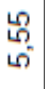 & $\stackrel{N}{N}$ \\
\hline ๔ฮ & $\begin{array}{c}\stackrel{\sim}{n} \\
m\end{array}$ & $\begin{array}{l}\overline{\tilde{j}} \\
\bar{m}\end{array}$ & ज्ञ & $\begin{array}{l}\circ \\
\infty \\
\infty \\
\infty\end{array}$ & $\begin{array}{l}m \\
\tilde{N} \\
\mathbb{J}\end{array}$ & $\begin{array}{l}\stackrel{m}{\sigma} \\
\stackrel{\sigma}{-}\end{array}$ & $\begin{array}{l}\text { : } \\
\stackrel{2}{0}\end{array}$ & $\begin{array}{l}\overline{9} \\
\text { 에 }\end{array}$ & $\begin{array}{l}\tilde{\infty} \\
\tilde{n} \\
\text { Na }\end{array}$ & $\begin{array}{l}\bar{\sigma} \\
\text { s. } \\
y\end{array}$ & 怘 & $\begin{array}{l}\text { 6. } \\
\stackrel{5}{2}\end{array}$ & $\begin{array}{l}\stackrel{8}{0} \\
\text { m. }\end{array}$ & \begin{tabular}{l}
$\infty$ \\
$\infty$ \\
\multirow{N}{*}{}
\end{tabular} & $\begin{array}{l}\bar{m} \\
\bar{m}\end{array}$ & $\stackrel{\delta}{\sigma}$ \\
\hline गे & $\underset{-}{f}$ & $\underset{N}{\mathbb{N}}$ & 희 & $\begin{array}{l}\infty \\
\stackrel{n}{N} \\
\text {. }\end{array}$ & $\sqrt[5]{2}$ & 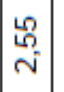 & 믜 & 희 & $\stackrel{\underset{1}{-}}{=}$ & 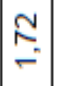 & $\begin{array}{l}0 \\
\tilde{m} \\
\tilde{\sigma}^{2}\end{array}$ & d & 寍 & $\stackrel{\mathbb{Z}}{\mathrm{J}}$ & $\bar{\infty}$ & $\begin{array}{l}\bar{\sigma} \\
\bar{\gamma}\end{array}$ \\
\hline 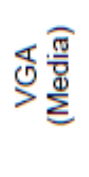 & 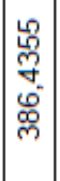 & \begin{tabular}{l}
2 \\
\multirow{6}{0}{} \\
0 \\
0
\end{tabular} & 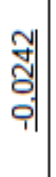 & 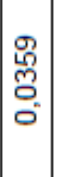 & $\begin{array}{l}\stackrel{N}{\circ} \\
\bar{\delta} \\
\delta^{\circ}\end{array}$ & \begin{tabular}{|l|}
$\frac{J}{\sigma}$ \\
$\frac{0}{0}$ \\
$\delta$
\end{tabular} & 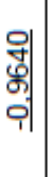 & $\left|\begin{array}{c}0 \\
\vdots \\
0 \\
0 \\
0 \\
\hdashline \\
7\end{array}\right|$ & $\begin{array}{l}\bar{\delta} \\
\bar{\delta} \\
\circ\end{array}$ & $\begin{array}{l}\overline{0} \\
\bar{\delta} \\
\overline{0}\end{array}$ & 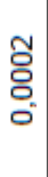 & $\begin{array}{l}\bar{\delta} \\
\overline{0} \\
0 \\
0\end{array}$ & 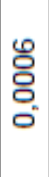 & 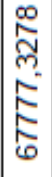 & 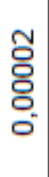 & 옹 \\
\hline$>\underbrace{\frac{\widehat{O}}{\mathrm{~g}}}$ & 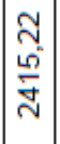 & 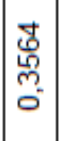 & 竞 & 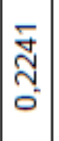 & $\begin{array}{l}\stackrel{2}{5} \\
\vdots \\
0 \\
\circ\end{array}$ & $\mid$\begin{tabular}{c|} 
\\
$\vdots$ \\
0 \\
0
\end{tabular} & 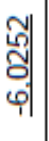 & 量 & 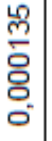 & $\mid \begin{array}{l}0 \\
0 \\
0 \\
0 \\
0 \\
0 \\
0\end{array}$ & 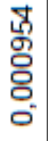 & $\begin{array}{l}\bar{\delta} \\
\text { оे } \\
\text { o. }\end{array}$ & 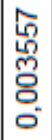 & 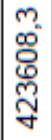 & 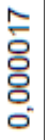 & 음 \\
\hline$\lessgtr \frac{\widehat{\underline{\underline{T}}}}{\mathrm{~d}}$ & 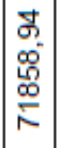 & $\mid \begin{array}{c}\mathbb{N} \\
\tilde{N} \\
\stackrel{N}{0} \\
0 \\
0\end{array}$ & 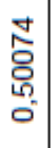 & $\mid \begin{array}{c}\mathcal{Z} \\
\mathcal{W} \\
\tilde{C} \\
0\end{array}$ & 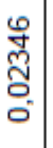 & 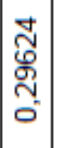 & 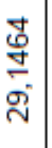 & 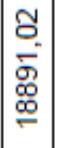 & 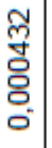 & 㐫 & 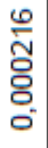 & 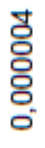 & 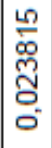 & 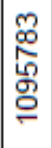 & 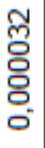 & $\begin{array}{l}\overline{3} \\
\text { 兽 } \\
0\end{array}$ \\
\hline 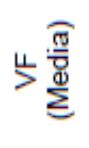 & 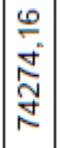 & 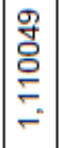 & 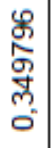 & $\mid \begin{array}{l}\mathscr{H} \\
\tilde{\infty} \\
\infty \\
0 \\
0 \\
0 \\
0\end{array}$ & 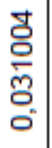 & 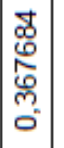 & 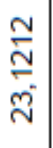 & 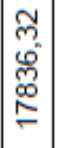 & 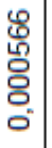 & $\begin{array}{l}\infty \\
\overline{0} \\
0 \\
0 \\
0\end{array}$ & $\begin{array}{l}\bar{\sigma} \\
\bar{\sigma} \\
0 \\
0\end{array}$ & 迢 & $\begin{array}{l}\mathcal{N} \\
\stackrel{N}{\widehat{J}} \\
0 \\
0\end{array}$ & $\begin{array}{l}\bar{\delta} \\
\bar{\sigma} \\
\overline{\underline{n}}\end{array}$ & 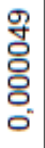 & $\bar{\delta}$ \\
\hline 岂す & 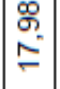 & $\begin{array}{l}\tilde{S}_{1} \\
\stackrel{n}{*}\end{array}$ & $\begin{array}{l}\text { స్ } \\
\text { के }\end{array}$ & S. & $\begin{array}{l}\stackrel{2}{2} \\
\stackrel{0}{-}\end{array}$ & $\begin{array}{l}\overline{\bar{\varphi}} \\
= \\
=\end{array}$ & $\begin{array}{c}\text { p. } \\
\text { స్ }\end{array}$ & $\left|\begin{array}{l}\stackrel{\infty}{\sim} \\
\mathbb{N}\end{array}\right|$ & 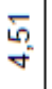 & $\left|\begin{array}{c}\mathcal{Y} \\
\underset{\forall}{*}\end{array}\right|$ & $\begin{array}{l}N \\
\omega^{\circ}\end{array}$ & $\begin{array}{l}\bar{\sigma} \\
\dot{f}\end{array}$ & $\frac{m}{\sigma}$ & $\begin{array}{l}\Omega \\
\infty \\
\infty\end{array}$ & 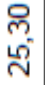 & $\begin{array}{l}\text { 怘 } \\
\text { - }\end{array}$ \\
\hline $\begin{array}{l}\frac{\mathrm{g}}{\mathrm{O}} \\
\frac{\mathrm{g}}{\mathrm{N}}\end{array}$ & 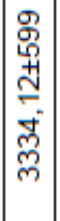 & 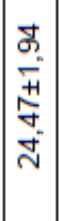 & 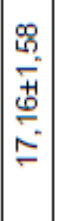 & 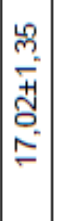 & 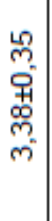 & 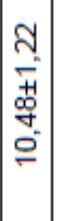 & 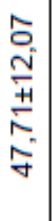 & 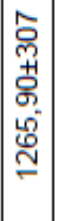 & 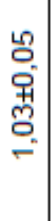 & $\mid \begin{array}{c}\overline{0} \\
0 \\
11 \\
0 \\
0 \\
0\end{array}$ & 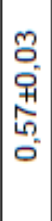 & 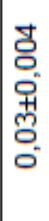 & 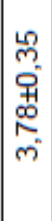 & 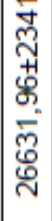 & 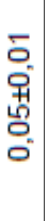 & 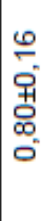 \\
\hline 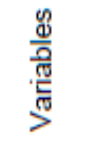 & $\begin{array}{l}\text { O्र } \\
\text { 䒘 }\end{array}$ & 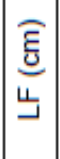 & 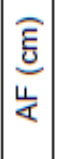 & 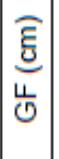 & 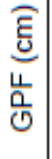 & 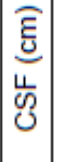 & 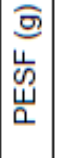 & 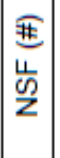 & 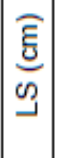 & \begin{tabular}{|l}
$\widehat{\hat{E}}$ \\
0 \\
0 \\
0
\end{tabular} & $\frac{\widehat{\bar{E}}}{\bar{c}}$ & $\begin{array}{l}\frac{\partial}{6} \\
\frac{0}{\alpha}\end{array}$ & $\begin{array}{l}\text { 울 } \\
0 \\
0 \\
\frac{0}{2}\end{array}$ & $\begin{array}{l}\text { 辇 } \\
\frac{\mathbf{L}}{\mathrm{N}} \\
\mathrm{Z}\end{array}$ & $\begin{array}{l}\overline{\bar{\xi}} \\
\stackrel{\infty}{>}\end{array}$ & 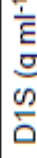 \\
\hline
\end{tabular}




\section{DISCUSIÓN}

Las diferencias significativas $(p<0,01)$ entre FHM para la característica AS, indica que al menos una de las FHM presentó promedios estadísticamente diferentes para el AS, más no así en PF, LF, AF, GF, GPF, CSF, PESF, NSF, LS, GS, P1S, P100S, NSK, V1S y D1S. Esta situación refleja poca variabilidad genética y alta homogeneidad fenotípica en las $10 \mathrm{FHM}$ evaluadas. Resultados contrarios se han reportado en la misma especie en varios estudios (Lovely y Vijayaraghava, 2017; Pradhan et al., 2018; Tadkal et al., 2019), donde se han detectado diferencias significativas al $5 \%$ y $1 \%$ entre genotipos para la mayoría de los caracteres del fruto y semillas considerados. Estos resultados opuestos a los obtenidos en esta investigación se pueden explicar posiblemente por el efecto fundador de una especie introducida al medio y acusa, por lo tanto, ausencia diversidad genética en la población estudiada (Resmi y Sreelathakumary, 2011; Matute at al., 2013) por alta endogamia y obliga a la introducción de nuevos cultivares para que al recombinarse con las poblaciones presentes, permitan el surgimiento de nuevas combinaciones genéticas favorables y con ello, una mejora sustancial en la población.

Los parámetros genéticos obtenidos para las características biométricas del fruto y semilla de $B$. hispida, con ligeras diferencias son similares a los rangos reportados por Lovely y Vijayaraghava, (2017); Pradhan et al. (2018); y Ekeke et al. (2019). Los caracteres biométricos GS, LS, AS, GF, LF, NSK, P100S y AF, sobresalieron por presentar menor dispersión y alta homogeneidad fenotípica, explicada por exhibir los más bajos coeficientes de variación fenotípica (CVF), a su vez las variables GPF, CSF, P1S y PF, presentaron dispersión media; mientras que D1S, NSF, PESF y V1S, exhibieron la mayor heterogeneidad y dispersión, explicada por los más altos CVF (Tabla 2). Estos resultados de CVF señalan rangos diferenciales de variabilidad fenotípica entre los caracteres estudiados, aunque sus valores en general muestren poca variabilidad para los caracteres más importantes a nivel comercial GPF y PF (Pradhan et al., 2018 y Ekeke et al., 2019).

Las estimaciones promedio de las VG, en general fueron bajas en todos los caracteres estudiados, excepto para el AS y GS de las semillas, en donde el componente genético representó más del $45 \%$ de la varianza fenotípica. Lo anterior, se correlacionó con altos valores de VA para la mayoría de los caracteres considerados en el estudio, esta situación permite inferir que las medias, VF y CVF estuvieron altamente influenciados por los efectos simples e interacciones de los factores bióticos y abióticos que actuaron sobre los genotipos, resultados similares han sido reportados en otros estudios (Kanimozhi et al., 2015; Lovely y Vijayaraghava, 2017; Pradhan et al., 2018). Se puede observar también que las magnitudes de las varianzas VG y VA, influyeron directamente en la magnitud de la VGA, CVG y los índices b en tales caracteres del fruto y las semillas (Nagaraju at al., 2016; Lovely y Vijayaraghava, 2017).

La comparación de los CVF de los 16 caracteres biométricos de los frutos y semillas, con sus correspondientes CVG contrastantes, se explica por altos efectos del ambiente (valores altos de VA media) y bajo o nulos efectos genéticos (VG y VGA) en la VF media, con valores de VG y VGA iguales negativos (asumidos como cero) o positivos, cercanos a cero para la mayoría de las características, exceptuando las características AS, D1S y P1S. Resultados similares han sido reportados por Kanimozhi et al. (2015), Nagaraju at al. (2016) y Lovely y Vijayaraghava (2017). Los bajos índices de b obtenidos para la mayoría de las características investigadas, corroboran la existencia de reducida variabilidad genética y el poco potencial de aprovechamiento de la varianza genética aditiva, excepto para el AS, que presentó el mayor valor $(0,93)$, lo cual la convierte en la variable con mayor probabilidad de progreso por selección. Estos resultados son opuestos a la importante diversidad genética reportada por Rabbani et al. (2013).

Se presentaron valores negativos en la VG y VGA para los caracteres AF, PESF y NSF (Tabla 3), esto claramente no debería presentarse, ya que, por ser expresiones cuadráticas, ninguna varianza puede ser negativa. Su origen se explica porque tales valores son estimados por substracción en donde el segundo término VA es mayor que el primero VF $(V G=V F-V A)$, situación similar ocurrió para VGA $(V G A=V G-$ VGNA). De acuerdo con Hallauer et al. (1988), las estimaciones de varianzas negativas son muy comunes en estudios de varianzas y componentes de varianzas en plantas, sugiriendo a su vez que las estimaciones de valores negativos puedan ser considerados y/o reportados como cero o valores negativos; por ello sugiere que ambas situaciones son válidas, que estos casos ocurren y que el investigador no debe pensar necesariamente que ha realizado algún cálculo en forma errónea o los resultados no tienen validez, por el sólo hecho de obtener un estimado de varianza negativo. Esta situación explica y permite comprender las estimaciones negativas e indefinidas en los otros parámetros que utilizan para sus cálculos a VG y VGA en las mismas variables.

De acuerdo con Kanimozhi et al. (2015), Nagaraju at al. (2016) y Lovely y Vijayaraghava (2017), los bajos CVF (<18\%) y CVG (<3\%) detectados en los 12 caracteres: GS, LS, AS, GF, LF, NSK, P100S, AF, GPF, CSF, P1S, PF y P1S, indican alta homogeneidad fenotípica y genética de los frutos y semillas en tales caracteres, hecho que reduce la posibilidad de lograr avance genético importante mediante la selección de 
plantas para estos caracteres, especialmente en el GPF y PF, los cuales son de mayor interés comercial en B. hispida (Pradhan et al., 2018; Tadkal et al., 2019). Esta situación muy posiblemente se deba a que la semilla de las FHM estudiadas, era proveniente de frutos de polinización cruzada entre pocas plantas y/o de una sola procedencia, con un origen genético común y alta endogamia, lo que se conoce en el mejoramiento genético de plantas como efecto fundador (Matute, 2013).

Los valores más altos obtenidos de heredabilidad en $\mathrm{h}^{2} \mathrm{~A}$ y $\mathrm{h}^{2} \mathrm{E}$ para el ancho de la semilla $(81,58 \%$ y $13,05 \%)$, permiten inferir que la selección basada en el fenotipo de este carácter es suficiente para lograr progreso genético, no obstante, los valores de $\mathrm{h}^{2} \mathrm{E}$, en todas las variables se clasificaron como de heredabilidad baja $(<20 \%)$. Estos resultados son inferiores a los reportados en otros estudios por Nagaraju at al. (2016), Lovely y Vijayaraghava (2017) y Tadkal et al. (2019), en los cuales se han reportado valores de heredabilidad en sentido amplio entre 50 a $80 \%$ y heredabilidad en sentido estricto entre 30 a $40 \%$ aproximadamente, para las características del fruto y semillas.

La ganancia genética esperada expresada como porcentaje de la media (GGE\%) importantes para AS y V1S, con aproximadamente un incremento en la GGE (\%) del $60 \%$ superior a sus respectivas medias, señalan un progreso genético importante en el mejoramiento de tales caracteres de la semilla de $B$. hispida. Estas ganancias genéticas esperadas por selección se consideran altas para estas dos características (GGE>20\%), mientras que las otras variables evaluadas, se agrupan como de baja GGE por selección, dado que no superaron a la media general. Ganancias genéticas esperadas (\%) superiores para estas características, han sido reportadas en otros estudios en la misma especie (Kanimozhi et al., 2015; Nagaraju at al., 2016; Lovely y Vijayaraghava, 2017), con incrementos del 20 al $60 \%$ con respecto a las medias para las características del fruto y semillas. Las estimaciones de los parámetros genéticos de las características AS, GS y V1S indican que son los más importantes para el mejoramiento genético de $B$. hispida, de modo que la selección de frutos más pesados con semillas anchas, gruesas y de mayor volumen, permitiría el mayor progreso genético y la obtención de plántulas vigorosas, como material de propagación, dado que la semilla constituye un insumo muy importante, en el establecimiento de un sistema de producción.

Por todo lo anterior, la determinación de las correlaciones genéticas y el análisis de sendero entre las características biométricas del fruto y semillas, además de la estrategia de aumentar la variabilidad genética por medio de intercambio, introducción y realización de cruzamientos entre germoplasma divergente, puede constituir el complemento ideal de un programa de mejoramiento genético en $B$. hispida en el Caribe húmedo colombiano, cuando el objetivo sea aumentar los caracteres biométricos de los frutos y semillas de forma sostenida (Lovely y Vijayaraghava, 2017; Pradhan et al., 2018).

\section{CONCLUSIONES}

De acuerdo con los resultados obtenido, discusión y comparación con los de otros autores, se pueden extraer las siguientes conclusiones: 1) Los estimadores de variabilidad genética resultaron importantes para las características, AS y GS de las semillas, en donde el componente genético representó el 81,58\% y 45,91\% de la varianza fenotípica; 2) Las heredabilidades más altas $h^{2} A=81,58 \%$ y $h^{2} E=13,05 \%$, fueron para AS, por lo que la selección basada en el fenotipo de este carácter es suficiente para lograr progreso genético; 3) La ganancia genética esperada expresada como porcentaje de la media, fue aproximadamente del $60 \%$, en el AS y V1S, lo que representa un progreso genético importante en el mejoramiento de tales caracteres de la semilla de B. hispida.

\section{AGRADECIMIENTOS}

Los autores desean agradecer el apoyo integral incondicional al Laboratorio de Genética Vegetal y Fitomejoramiento (LAGEFI) de la Facultad de Ciencias Agrícolas de la Universidad de Córdoba (Montería Colombia), en la realización del trabajo.

\section{REFERENCIAS}

Al-Snafi, A.E., The Pharmacological Importance of Benincasa hispida (Thunb.) Cogn. A Review. Int J Pharm Sci Res., 4(12), 165-170 (2013).

Araméndiz-Tatis, H., Cardona-Ayala, C.E y Combatt-Caballero, E.M., Contenido Nutricional de Líneas de Fríjol Caupí (Vigna unguiculata L. Walp.) Seleccionadas de una Población Criolla, doi: 10.4067/S0718-07642016000200007, Inf. tecnol., 27(2), 53-60 (2016).

Chakraborty, K., Datta, M., y otros tres autores., Hypoglycemic Activity of the Fruits of Benincasa hispida (Thunb.) Cogn. Found in State of Tripura, India. ISSN 0975-413X, Der Pharma Chem., 10(8), 102-104 (2018). 
Chomicki, G., Schaefer, H., y Renner, S.S., Origin and Domestication of Cucurbitaceae Crops: Insights from Phylogenies, Genomics and Archaeology, https://doi.org/10.1111/nph.16015, New Phytol., 226(5), 1240-1255 (2020).

Cruz, C.D., Programa Genes V.2014.6.1 - Aplicativo Computacional em Genética e Estatística, http://www.ufv.br/dbg/genes/genes.htm, Consultado: 22 septiembre (2016).

Ekeke, C., Ogazie, C.A., y Agbagwa, I.O., Anatomical and Phytochemical Studies on Benincasa hispida (Thunb.) Cogn. (Cucurbitaceae), https://doi.org/10.15835/nsb11110394, No Sci Biol., 11(1), 102-111 (2019).

Estrada, H.H., Restrepo, C.E., y otros dos autores., Deshidratación Osmótica y Secado por Aire Caliente en Mango, Guayaba y Limón para la Obtención de Ingredientes Funcionales, http://dx.doi.org/10.4067/S0718-07642018000300197, Inf. tecnol., 29(3), 197-204 (2018).

Gupta, P., Chikkala, C., y Kundu, P., Ash Gourd and its Applications in the Food, Pharmacological and Biomedical Industries, https://doi.org/10.1080/19315260.2019.1699222, Int J Veg Sci., 1(1), 1-10 (2019).

Hallauer, A.R., Carena, M.G., y Miranda, F.J.B., Quantitative Genetics in Maize Breeding, 2a Ed; 169-184: lowa State Univ. Press, lowa, USA (1988).

Jiang, X., Kuang, F., Kong, F., y Yan, C., Prediction of the Antiglycation Activity of Polysaccharides from Benincasa hispida (Thunb.) Cogn. Using a Response Surface Methodology, https://doi.org/10.1016/j.carbpol.2016.05.079, Carbohydr Polym., 151(1), 358-363 (2016).

Kanimozhi, R., Mohammed Y.G., y otros tres autores., Genetic Analysis in Segregating Generation of Wax Gourd, https://doi.org/10.1080/19315260.2013.876568, Int J Veg Sci., 21(3), 281-296 (2015).

Lovely, B., y Vijayaraghava, K., Estimates of Genetic Variability, Heritability and Genetic Advance for Yield and Yield Component Traits in Ash Gourd [Benincasa hispida (Thunb.) Cogn.] Genotypes, Doi: 10.18805/ag.D-4676, Agric Sci Digest., (37), 270-274 (2017).

Matute, D.R., The Role of Founder Effects on the Evolution of Reproductive Isolation, https://doi.org/10.1111/jeb.12246, J Evol Biol., 26(11), 2299-2311 (2013).

Nagaraju, K., Saraswati, T., y otros tres autores., Study on Variability and Genetic Parameters in Ash Gourd [Benincasa hispida (Thunb.) Cogn.] Genotypes, Doi: 10.24247/ijasrjun2016019, Int J Agric Sci Res., 6(3), 147-162 (2016).

Palencia, G.; Mercado, T., y Combatt, E., Estudio Agroclimático del Departamento de Córdoba, 1er Ed; 37-42: Facultad de Ciencias Agrícolas, Universidad de Córdoba, Montería, Colombia (2006).

Pandey, A.K., Bhardwaj, D.R., y otros tres autores., Botany, Diversity, Utilization And Improvement of Ash Gourd [Benincasa Hispida (Thunb.) Cogn] - A Review, Ann. Hort., 8(1), 1-15 (2015).

Pradhan, K., Nandi, A., y otros cuatro autores., Genetic Variability and Varietal Performance in Ash Gourd [Benincasa hispida (Thunb.) Cogn.] Genotypes, ISSN (Online): 0973-7049, The Bioscan., 13(2), 791-794 (2018).

Pradhan, K., Nandi, A., Rout, S., y Tripathy, B., Ash Gourd - an Under Exploited Potential Crop, https://doi.org/10.20546/ijcmas.2018.703.258, Dogo Rangsang Res J., 10(06), 142-151 (2020).

Rabbani, M.G., Naher, U.A., Pramanik, S., y Quais, M.K., Genetic Diversity of Ash Gourd [Benincasa hispida (Thunb.) Cogn.] Genotypes, https://doi.org/10.3329/bjpbg.v26i2.23842, Bangladesh J Plan Breed Gen., 26(1), 01-08 (2013).

Resmi, J., y Sreelathakumary, I., Genetic Variability and Character Associations in Ash Gourd [Benincasa hispida (Thunb.) Cogn.], ISSN (Online): 0976-0547, Agric Sci Digest., 31(3), 193-197 (2011).

Rodríguez-Manrique, J.A., Alvis-Bermudez, A., y Cohen-Manrique, C.S., Análisis de Perfil de Textura de Ahuyama (Cucurbita maxima) sometida a Freído Atmosférico por Inmersión, http://dx.doi.org/10.4067/S0718-

07642018000400055, Inf. tecnol., 29(4), 55-64 (2018).

Sureja, A.K., Sirohi, P.S., Patel, V.B., y Mahure, H.R., Estimation of Genetic Parameters in Ash Gourd, ISSN (Online): 0974-0112, Indian J Hortic., 67(1), 170-173 (2010).

Tadkal, R., Beaulah, A., Krishnamoorthy, V., y Thangaraj, K., Evaluation of Ash Gourd [Benincasa hispida) (Thunb.) (Cogn.] Genotypes for Growth and Yield Under Pandal System of Cultivation, E-ISSN: 2321-4902, Int J Chem Stud., 7(3), 2933-2937 (2019).

Tasiguano, B.L., Villarreal, C., Schmiele, M., y Vernaza, M.G., Efecto del tiempo de Cocción del Zapallo (Cucurbita maxima) y la adición de Glucosa Oxidasa en el Aumento de Almidón Resistente del Pan de Molde, http://dx.doi.org/10.4067/S0718-07642019000300167, Inf. tecnol., 30(3), 167-178 (2019). 
